\title{
COVID-19 desde una óptica tecno-educativa a través de markerspaces
}

\section{COVID-19 from a techno-educational perspective through markerspaces}

Noelia Margarita Moreno Martínez.

Universidad de Málaga. nmarg@uma.es

María Belén Morales-Cevallos. Universidad Católica de Santiago de Guayaquil (Ecuador). mabelenmorales0@gmail.com

\section{RESUMEN.}

Ante la emergencia sanitaria acontecida a nivel mundial debido a la pandemia de COVID-19, en el ámbito educativo surge la necesidad de diseñar material didáctico para dar a conocer al alumnado dicho fenómeno y las mejores formas de actuar propiciando actitudes solidarias, un trabajo comunitario conjunto y una conciencia social. Para ello, en este trabajo se presentan herramientas de realidad aumentada, realidad virtual, modelado en 3D e impresión en 3D para favorecer el estudio, el análisis y la comprensión de la naturaleza, la estructura, la composición y el origen del virus, los factores que desencadenan los contagios, la sintomatología que presenta el sujeto infectado, fórmulas de actuación par parte del personal sanitario en los hospitales y las medidas de prevención y seguridad. Desde un enfoque innovador el profesorado de la era digital debe poseer una formación desde el punto de vista pedagógico e instrumental y tecnológico para acometer con acierto su labor docente orientada a facilitar la adquisición y construcción del conocimiento por parte de su alumnado. Para ello, se considera de especial importancia el soporte, el material empleado como mediador, en este caso las tecnologías emergentes para propiciar el aprendizaje en red desde una perspectiva conectivista atendiendo a la filosofía colaborativa y creativa del makerspaces.

\section{PALABRAS CLAVE.}

Tecnologías emergentes; Realidad aumentada; Realidad virtual; Modelado en 3D; Impresión en 3D; Makerspaces.

\section{ABSTRACT.}

In the face of the health emergency that occurred worldwide due to the COVID-19 pandemic, in the educational field there is a need to design teaching materials to make this phenomenon known to students and the best ways to act promoting solidarity attitudes, joint community work and a social conscience. To do this, this work presents augmented reality tools, virtual reality, 3D modeling and 3D printing to promote the study, analysis and understanding of the nature, structure, composition and origin of the virus, the factors that They trigger infections, the symptoms presented by the infected subject, formulas for action by health personnel in hospitals, and prevention and safety measures. From an innovative approach, teachers of the digital age must have training from the pedagogical and instrumental and technological point

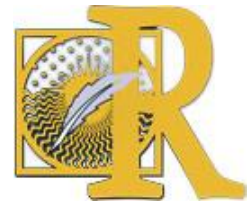


of view to successfully undertake their teaching work aimed at facilitating the acquisition and construction of knowledge by their students. For this, the support, the material used as a mediator, in this case the emerging technologies to promote online learning from a connectivist perspective, considering the collaborative and creative philosophy of makerspaces, is considered of special importance.

\section{KEY WORDS.}

Emerging technologies; Augmented reality; Virtual reality; 3D modeling; 3D printing; Makerspaces.

\section{Introducción y análisis epistemológico conceptual: realidad aumentada, modelado en 3D e impresión en 3D para el aprendizaje}

Ante los recientes acontecimientos que estamos viviendo tan cambiantes y ambivalentes cada vez queda más patente la revolución que está suponiendo la incorporación de las TIC en todos los campos profesionales en lo relativo a la renovación de los procesos de trabajo desde un enfoque colaborativo, una comunicación más fluida de forma sincrónica y asincrónica y una formación continua para el desarrollo de competencias digitales (Vázquez, León, Parra y López-Meneses, 2020). Nuestras vidas son digitalizadas y digitales (López y Bernal, 2019) lo que requiere de una ciudadanía formada adecuadamente para poder afrontar retos, desafíos y novedades que trae consigo la sociedad del conocimiento y de la información. Nunca antes a lo largo de su historia, la humanidad había tenido a su disposición tantas TIC como en la actualidad, tecnologías que se duplican a gran velocidad gracias a la digitalización, haciendo que lo transmedia, es decir, la observación de los contenidos mediáticos en diferentes dispositivos, multiplique las formas en las cuales podemos interaccionar las personas con la información (Cabero y Ruiz, 2018). Así pues, en el presente trabajo se pretende dar a conocer las ventajas de tecnologías emergentes como la realidad aumentada, programas de modelado en 3D desde una perspectiva de makerspaces al profesorado, al alumnado y a la comunidad educativa en general como herramientas didácticas para la enseñanza del fenómeno de la pandemia de COVID19 y para propiciar actitudes responsables y solidarias orientadas hacia la seguridad y la protección para hacer frente a la propagación del virus.

\subsection{Realidad aumentada.}

La realidad aumentada $(R A)$ es una tecnología que nos permite la visualización directa o indirecta de elementos del mundo real combinados (o aumentados) con elementos virtuales generados por un ordenador, cuya fusión da lugar a una realidad mixta (Cobo y Moravec, 2011). En la misma línea Azuma (1997) la concibe como aquella tecnología que combina elementos reales y virtuales creando escenarios interactivos en tiempo real y registrados en 3D. También es definida como aquel entorno en el que tiene lugar la integración de lo virtual y lo real (Cabero y García, 2016; Cabero y Barroso, 2016a, 2016b; Cabero, Leiva, Moreno, Barroso y López_Meneses, 2016; Moreno, Leiva y López-Meneses, 2017; Moreno y Leiva, 2017; Tecnológico Monterrey, 2017, Moreno, López-Meneses y Leiva, 2018a, 2018b). Por lo tanto, la tecnología de realidad aumentada nos permite complementar, amplificar, enriquecer y potenciar nuestro entorno circundante real añadiendo capas de información digital y

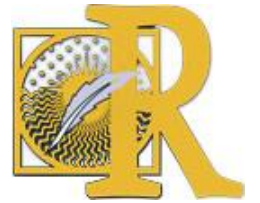


modelos tridimensionales que constituyen esa fuente de información adicional que permite el análisis, la interpretación y la comprensión de fenómenos complejos o difíciles de obtener para mostrarlos en un escenario real.

\subsection{Realidad virtual}

La realidad virtual se define como un sistema informático que genera fundamentalmente una simulación y representación computarizada de la realidad. Es decir, la realidad virtual se caracteriza por su naturaleza inmersiva como aquella tecnología que posibilita al usuario, mediante el uso de un visor de RV, sumergirse en escenarios tridimensionales en primera persona y en 360 grados (Nugent, 1991; Casey, 1994; Auld, 1995; Moreno y Ramírez, 2016; Moreno, Leiva y Mac, 2017; Moreno, López-Meneses y Leiva, 2018b). Según Vera, Ortega y Burgos (2003) entre las necesidades, condiciones o requisitos que debe cumplir una instalación de realidad virtual para poder reconocerla como tal, destacan las siguientes: Simulación: Capacidad para representar un sistema con suficiente parecido a la realidad, para convencer al usuario de que constituye una situación paralela a aquella. Este entorno estará regido por una serie de reglas, no necesariamente iguales a las del mundo real; Interacción: Tener el control del sistema creado para que las acciones del usuario produzcan cambios en el mundo artificial. Para lograr esta interacción existen diversos interfaces hombre-máquina, que van desde los más sencillos como teclado y ratón hasta otros más avanzados como guantes o trajes sensoriales; y Percepción: Es el factor más importante de todos. Actualmente los sistemas de Realidad Virtual se dirigen principalmente a los sentidos (vista, oído, tacto) mediante elementos externos (lentes y cascos de visualización o HMD, guantes de datos, etc).

\subsection{Modelado en 3D.}

En el lenguaje de los gráficos en 3D, un modelo es un archivo que contiene la información necesaria para ver o "renderizar" un objeto en tres dimensiones (Moreno, López-Meneses y Leiva, 2018a). Este archivo contiene dos tipos de información: 1. La geometría, que hace referencia a la forma del objeto (esfera, cubo, cilindro, cono, donut, prisma, semiesfera, pirámide). Para el sistema computacional, la información de la geometría del modelo define las superficies del objeto como una lista de polígonos planos que comparten lados y vértices. El modelo se diseña sobre una malla. 2. Los atributos de la superficie del objeto, son aquellas características que definen la apariencia del objeto en cuanto a color y textura con el objetivo de atribuirle el máximo realismo y parecido con el elemento que representa en cuanto al material del que está hecho.

\subsection{Impresión en 3D.}

La impresora en 3D es una máquina que nos permite realizar impresiones de objetos 3D a partir de un diseño o prototipo concreto en formatos como: .obj, .stl, .fbx generado mediante un programa CAD (de diseño asistido por ordenador) o plataformas y programas de modelado en 3D como 3DC: https://3dc.io/app/ , Tinkercad: https://www.tinkercad.com o Blender entre otros. Dichos diseños en 3D pueden ser piezas, figuras básicas o primitivas, maquetas (Moreno, Leiva y López-Meneses, 2016; Beltrán y Rodríguez, 2017; Moreno, López-Meneses

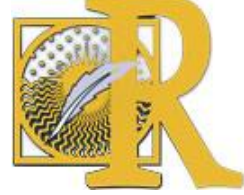


y Leiva, 2018a). Los sectores en los que comenzaron a utilizarse son en arquitectura y en diseño industrial. En la actualidad se está extendiendo su uso en la fabricación de prótesis médicas, ya que la impresión 3D permite adaptar cada pieza fabricada a las características exactas de cada paciente. Aunque cada vez en mayor medida, se utilizan estas impresoras en el contexto educativo como recurso didáctico.

\subsection{Makerspaces.}

Los makerspaces constituyen espacios abiertos, dinámicos e interactivos para la invención y la fabricación digital creativa haciendo posible que los usuarios sean productores activos de contenido para la comunidad desde una concepción de democratización del conocimiento para lograr una transformación social a través de la innovación y el desarrollo tecnológico. Así pues, según Smith (2017) los makerspaces se conciben como espacios de emprendedores basados en la comunidad y para la democratización de la actividad donde los usuarios tienen acceso a las herramientas, las habilidades y los colaboradores expertos con la finalidad de construir el conocimiento desde un enfoque colaborativo para el enriquecimiento mutuo del colectivo implicado.

Los defensores de los makerspaces para la educación destacan el beneficio de los estudiantes que participan en la resolución de problemas creativos de orden superior a través de un acercamiento práctico al diseño, la construcción y la interacción. La cuestión de cómo renovar o reutilizar las aulas para hacer frente a las necesidades del futuro se está respondiendo a través del concepto de los makerspaces o talleres que ofrecen herramientas y las experiencias de aprendizaje necesarias para ayudar a las personas a llevar a cabo sus ideas (Horizon Report, 2015, 2018). En la misma línea, Portuguez y Gómez (2019) los consideran como espacios de educación no formal donde los participantes utilizan las herramientas tecnológicas puestas al servicio de la comunidad para diseñar prototipos, experimentar con nuevos materiales, imprimir modelos 3D y buscar alternativas para mejorar o inventar productos innovadores.

\section{Herramientas de realidad aumentada para el estudio del fenómeno relacionado con la pandemia de COVID-19 desde una perspectiva didáctica.}

En este apartado se presentan las herramientas de realidad aumentada que tanto el profesorado como el alumnado tienen a su disposición desde dispositivos móviles y tablets para propiciar escenarios de aprendizaje mixtos en los que la información del contexto físico real se amplifica con modelos 3D que representan el objeto de estudio.

\subsection{JigSpace.}

Es un aplicación móvil que nos permite visualizar escenarios de realidad aumentada relacionados con diferentes contenidos didácticos. En la figura 1 se muestra una captura de pantalla de la librería o galería de la aplicación con algunas escenas didácticas sobre diversas disciplinas. Esta aplicación está disponible en la plataforma App Store para iPhone y iPad. En dispositivos Android se puede obtener descargando el apk desde esta web: https://apkpure.com/es/jigspace/com.JigSpace.JigViewer ya que no se encuentra en la plataforma Play Store.

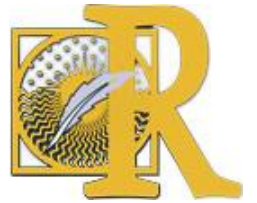




\section{INTERNATIONAL JOURNAL OF EDUCATIONAL}
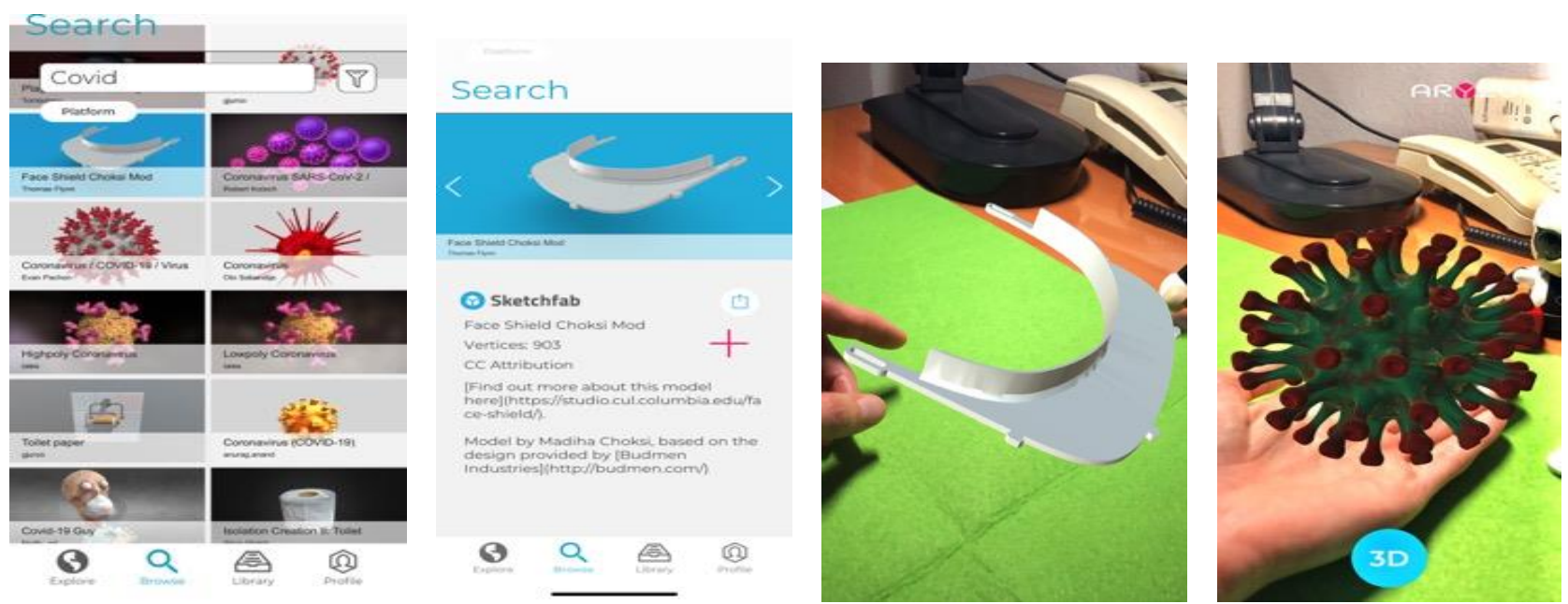

Figura 3. Galería de objetos 3D Sketchfab accesible desde la aplicación móvil AR Studio. Figura 4. Prototipo de objeto 3D de una visera protectora facial como material sanitario aportado por el diseñador Thomas Flynn disponible para su descarga en formato .stl desde la galería Sketchfab desde este enlace:

https://skfb.ly/6Rpru. Figura 5. Modelo 3D de la visera protectora facial proyectada en el escenario real con la aplicación AR Studio. Figura 6. Escenarios de RA creado con la aplicación AR Studio para la visualización del virus COVID-19. Fuente: Elaboración propia empleando la aplicación móvil AR Studio y los modelos en 3D de

\subsection{Object Viewer.} la galería Sketchfab.

La aplicación móvil Object Viewer nos permite proyectar sobre el cubo Merge modelos 3D (Figura 7, Figura 8 y Figura 9) que podemos obtener de la galería de la propia aplicación o bien de galerías de objetos tridimensionales como Google Poly o Sketchfab que hayamos descargado e importado en la aplicación. Dicho cubo lo podemos obtener a través del siguiente enlace: https://mergeedu.com/download/file.php?f=paper-merge-cube.pdf

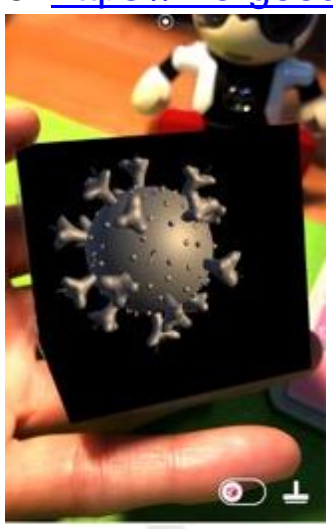

SARS_CoV_2-lowpoly

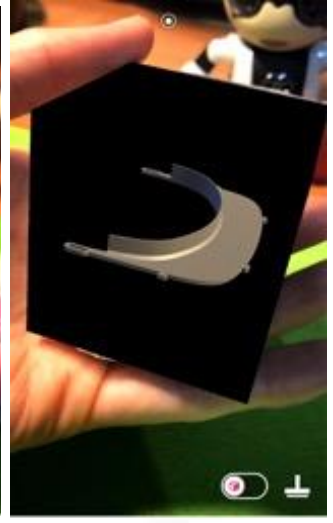

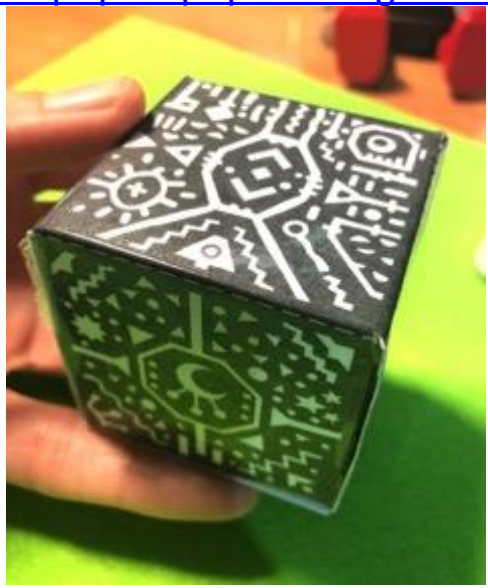

Figura 7. Muestra de modelo en 3D del COVID-19 proyectado sobre el cubo Merge para su visualización en realidad aumentada. Figura 8. Muestra de modelo en 3D de visera protectora facial proyectada sobre cubo Merge para su visualización en realidad aumentada. Figura 9. Vista de cubo Merge. Fuente: Elaboración propia empleando la aplicación móvil Object Viewer, los modelos en 3D de la galería Sketchfab y el cubo Merge. 


\section{Realidad virtual para la capacitación del personal sanitario para hacer frente a la situación de COVID-19 en el ámbito hospitalario}

Las empresas estadounidenses de salud Shift y Cleanbox con el apoyo de la Fundación Facebook y la Universidad de Portland se han unido para desarrollar un programa de capacitación sobre el COVID-19 en realidad virtual que consiste en el entrenamiento del personal sanitario en escenarios inmersivos que simulan la propia realidad en sesiones que duran entre 15 y 20 minutos sobre el cuidado e higiene del paciente y los procedimientos de prueba COVID-19. De este modo, a través de estas experiencias inmersivas se puede constatar las potencialidades de la tecnología de realidad virtual al servicio de la capacitación y el entrenamiento de los trabajadores de la salud dotándolos de conocimientos a nivel procedimental para acometer situaciones de alto riesgo sin exponerlos a los peligros que supone enfrentarse a entornos reales sin previa formación. En la figura 10 se muestra un ejemplo de escenario de realidad virtual que representa la situación de capacitación del personal sanitario ante el COVID-19 en el ámbito hospitalario.

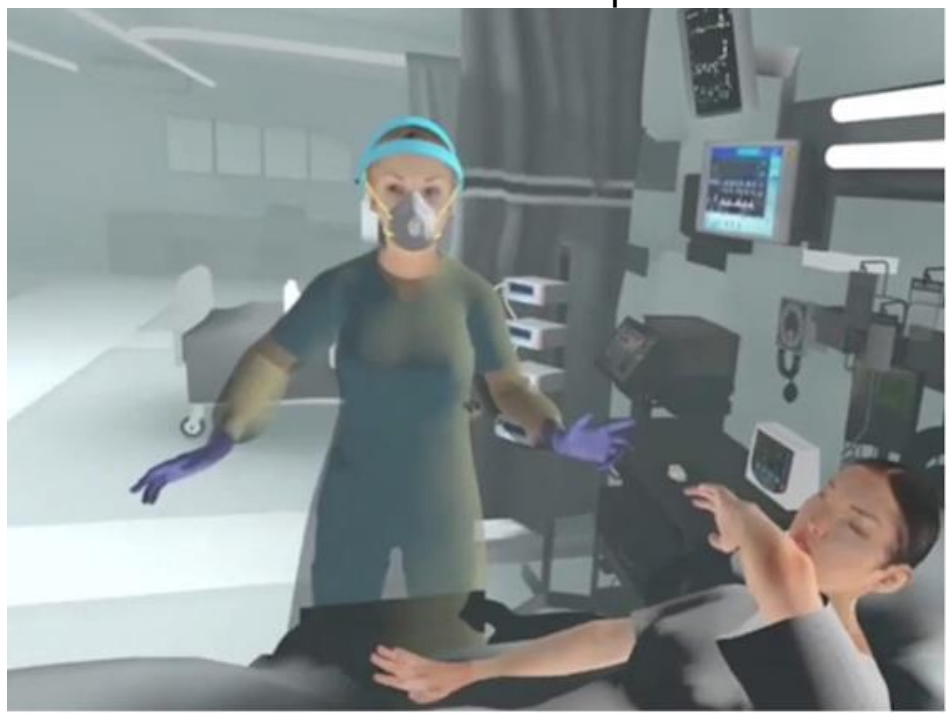

Figura 10. Muestra de escenario de realidad virtual para la capacitación del personal sanitario en el ámbito hospitalario ante el COVID-19. Fuente: Umbral Virtual: http://umbralvirtual.com/2020/05/28/capacitacion-enrealidad-virtual-sobre-el-covid-

19/?fbclid=IwAR2IKHkzCfUhghsAB7mYdGLXnenFt6DNw69HQdGkbhOrkkwjkrqV0aY0d3A

\section{Herramientas de modelado en 3D e impresión en 3D para el estudio del fenómeno relacionado con la pandemia de COVID-19 desde una perspectiva didáctica.}

A continuación, se presentan por un lado, plataformas y programas de modelado en 3D para el diseño de prototipos que podemos imprimir en formato físico con impresoras en 3D o bien proyectar en el contexto físico real generando escenarios de realidad aumentada. Y por otro lado, galerías de objetos 3D para visualizar y para descargar modelos tridimensionales que podemos modificar en plataformas y programas de modelado en 3D, proyectar en el contexto real o imprimir en formato manipulativo con impresoras en 3D.

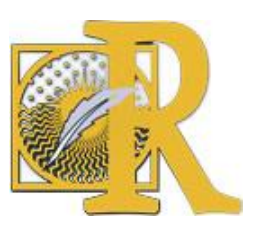




\subsection{Plataformas y programas de modelado en $3 D$.}

- 3DC: es una herramienta de modelado en 3D disponible como plataforma web online y aplicación móvil para smartphone y tablets. Para acceder a esta plataforma se aporta el siguiente enlace: https://3dc.io

- Tinkercad: es una plataforma desarrollada por Autodesk para la creación y modelado de objetos 3D. Para acceder a esta plataforma se aporta el siguiente enlace: https://www.tinkercad.com . La figura 11 muestra un ejemplo de creación de objetos 3D con la herramienta Tinkercad para familiarizar al usuario con la interfaz de diseño, las opciones (girar sobre el propio eje, rotar, ampliar, reducir, mover, etc; importar, exportar o guardar figuras en formato .obj 0 .stl) y las piezas para realizar el modelado en 3D.

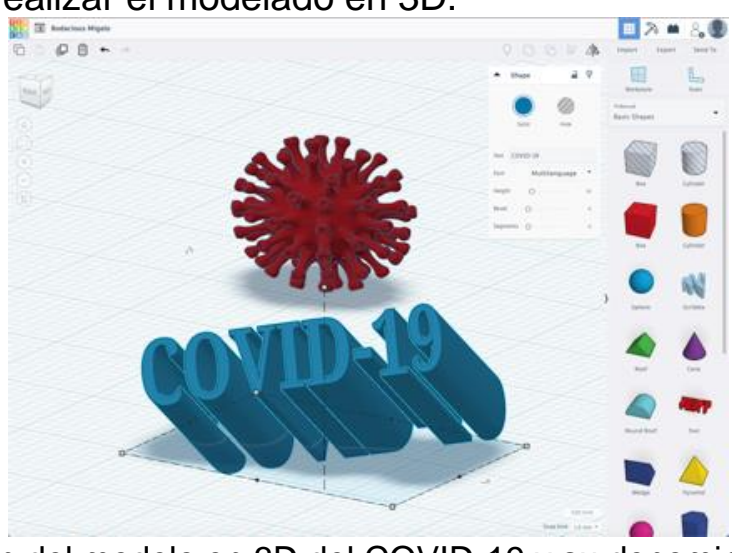

Figura 11. Muestra de creación del modelo en 3D del COVID-19 y su denominación empleando texto en 3D.

Fuente: Elaboración propia con la plataforma de modelado Tinkercad.

- Blender: es un programa informático multiplataforma, dedicado especialmente al modelado, animación y creación de gráficos tridimensionales. El programa fue inicialmente distribuido de forma gratuita, pero sin el código fuente, con un manual disponible para la venta, aunque posteriormente pasó a ser software libre. Actualmente es compatible con todas las versiones de Windows, Mac OS X, GNU/Linux, Solaris, FreeBSD e IRIX. Disponible para su descarga en: https://www.blender.org/ Manual de uso: http://www.ite.educacion.es/formacion/materiales/181/cd/indice.htm

- SketchUp: Es un programa de diseño gráfico y modelado en 3D basado en caras, fue desarrollado por @Last Software, empresa adquirida por Google en 2006 y nació como complemento de Google Earth hasta que finalmente fue vendida a Trimble Buildings en 2012. Este programa actualmente no sólo es utilizado por profesionales, sino también por estudiantes y profesores. El objetivo de esta aplicación es que a través de sus diferentes productos los estudiantes puedan utilizarlos para expresar ideas, descubrir nuevas posibilidades artísticas y fomentar la creatividad. Posee diferentes tutoriales así como asesoramiento para su aplicación en centros educativos en diferentes países del mundo. Disponible en: http://www.sketchup.com/es

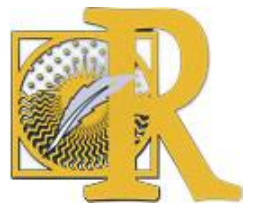




\subsection{Galerías de objetos tridimensionales.}

Además de los programas mencionados para crear nuestros propios modelos tridimensionales, disponemos de galerías de modelos tridimensionales gratuitos cuyos objetos 3D están clasificados por categorías para su descarga y posterior impresión con impresoras 3D o bien para proyectarlos en el contexto real con tecnología de realidad aumentada empleando aplicaciones móviles como AR Viewer, AR Studio, Object Viewer entre otras.

- 3D Warehouse: https://3dwarehouse.sketchup.com/?hl=es

- $\quad$ Archive 3D: https://archive3d.net

- Sketchfab: https://sketchfab.com/feed

- Google Poly: https://poly.google.com

\subsection{Impresión en 3D para fabricar material sanitario: máscaras o viseras de protección facial, salvaorejas en 3D, respiradores y mascarillas en 3D}

Ante el aumento masivo de infecciones de coronavirus y la escasez de materiales en el ámbito sanitario, surgen iniciativas solidarias y altruistas desde la empresa privada, desde particulares e ingenieros de centros de investigación para fabricar máscaras o viseras de protección facial, salvaorejas en 3D, respiradores y mascarillas en 3D.

\subsubsection{Impresión de máscaras o viseras de protección facial en 3D.}

Para la impresión en 3D de máscaras o viseras de protección facial en 3D, desde la iniciativa particular y unidos al movimiento coronavirus_makers siguiendo el patrón establecido por un grupo de italianos que diseñaron el prototipo inicialmente disponible para su descarga e impresión usando impresoras en 3D través de este enlace: https://lanpdt.life/upshield En la Figura 12 se muestra el prototipo de modelo en 3D de la visera protectora facial, a la cual una vez impresa en formato físico, se le incorpora una lámina o pantalla protectora de acetato y una cinta elástica para sostener la visera en la cabeza:

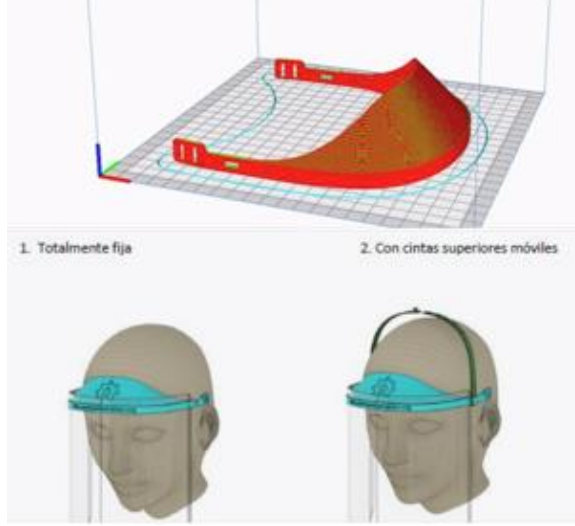

Figura 12. Prototipo de modelo en 3D de visera de protección facial. Fuente: UPShield Face Shield Mask.

A partir del prototipo inicial, surgen más creaciones entre las que se muestran a continuación: - Prototipo 1 de visera protectora facial en formato .stl aportado por el diseñador Thomas Flynn (Figura 13). A través de este enlace se puede acceder a dicho prototipo en formato .stl ubicado en la galería Sketchfab para su descarga e impresión en 3D: https://sketchfab.com/3d-models/face-shield-choksi-mode43ded4230c0458093b275a5ece52973

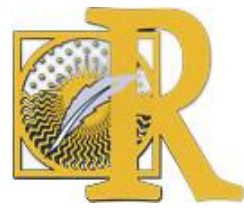




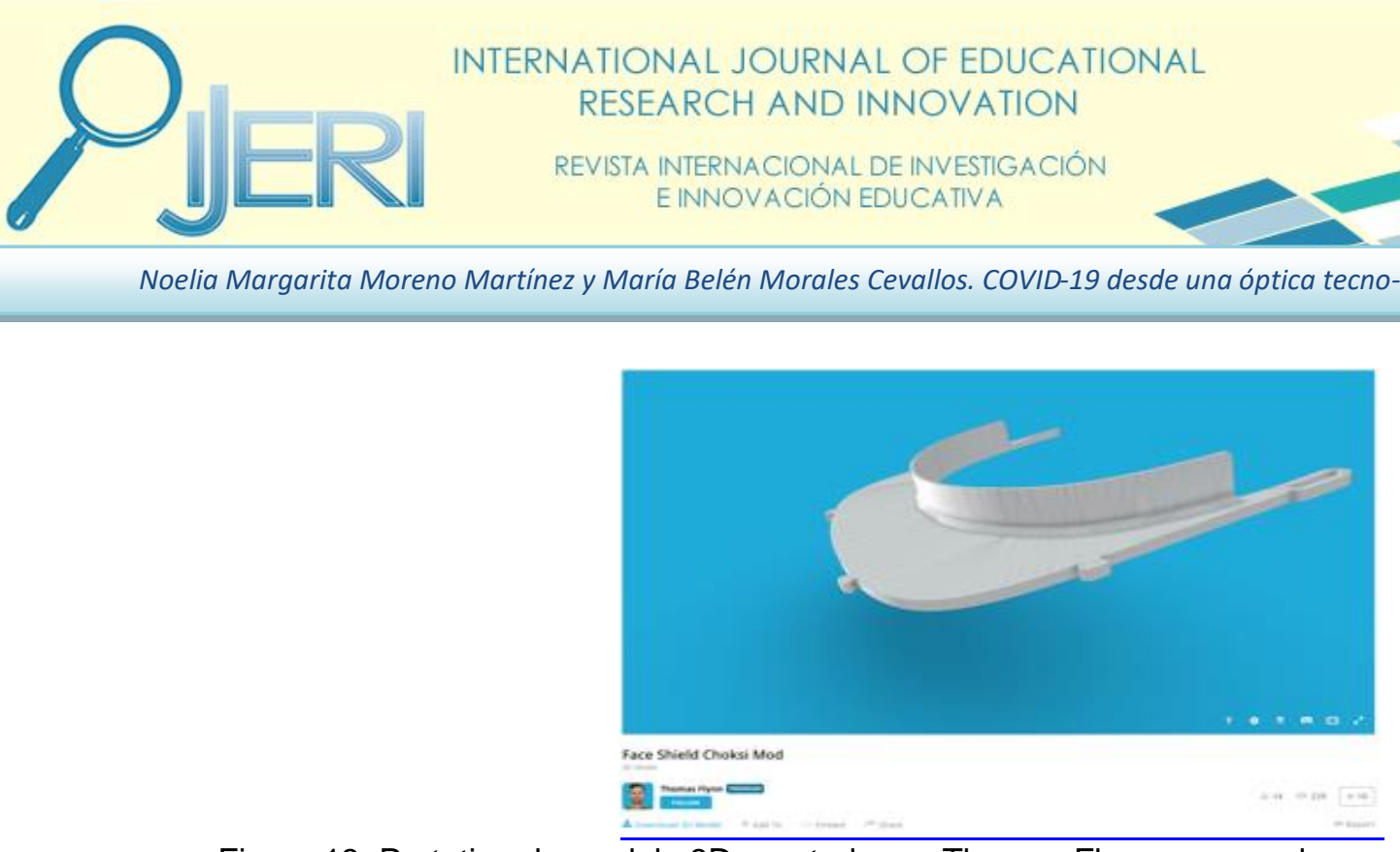

Figura 13. Prototipo de modelo 3D aportado por Thomas Flynn para su descarga en formato .stl desde la galería de objetos 3D Sketchfab. Fuente: Thomas Flynn.

- Prototipo 2 de visera protectora facial aportado por el equipo del Museo de Historia Natural de la Plata, Argentina obtenido de la comunidad virtual Cults. A través de este enlace se puede acceder a este prototipo en formato .stl para su descargar e impresión en 3D: https://sketchfab.com/3dmodels/visera-plana-23badf4979e744d7b7ace8436831d2af

- Prototipo 3 de visera protectora facial aportado por un diseñador de Induna-Art-Design. A través de este enlace se puede acceder a este prototipo en formato .stl para su descarga e impresión en 3D:

https://sketchfab.com/3d-models/induna-ppe-face-sheild-3d-printable-versioncc287f67a19344cd8eb4b811472fe1cc

\subsubsection{Impresión de salvaorejas en 3D.}

Para evitar las molestias y el daño que pueden causar en las orejas las gomas elásticas de las mascarillas después de muchas horas, surge inicialmente a petición del personal sanitario la necesidad de fabricar piezas en 3D denominadas salvaorejas para mascarillas. Y ante esta demanda, bajo la iniciativa de makers comprometidos para dar una respuesta eficaz, diseñan esta pieza en 3D (Figura 14), la cual se puede descargar en formato .stl a través del siguiente enlace: https://www.thingiverse.com/thing:4249113

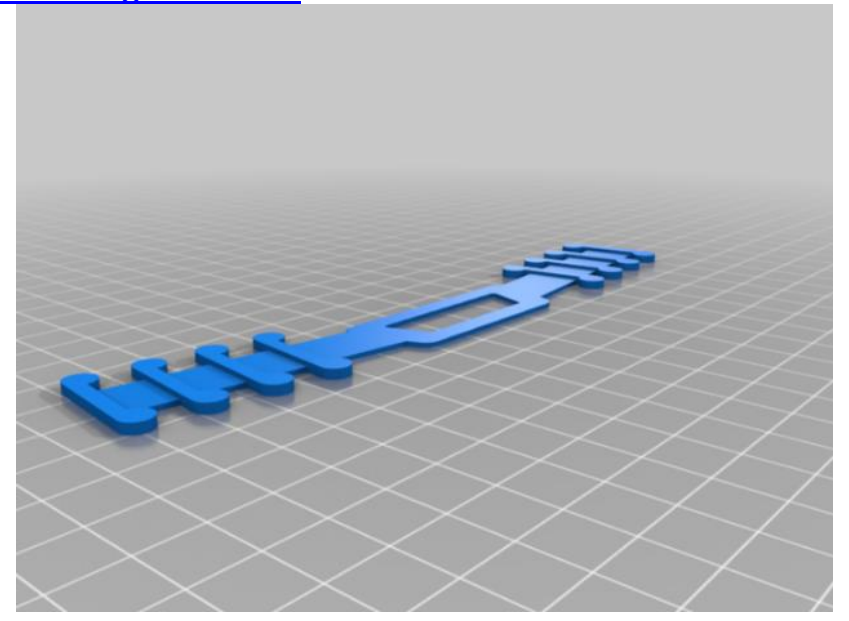

Figura 14. Pieza en 3D de salvaorejas. Fuente: diseñador Suraky: https://www.thingiverse.com/Suraky/about

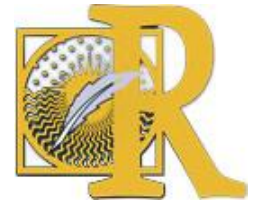




\subsubsection{Impresión de piezas en 3D para convertir máscaras de buceo en respiradores.}

El equipo de ISINNOVA aporta una pieza en 3D para adaptar las máscaras de buceo snorkel y convertirlas en respiradores (Figura 15). En el siguiente enlace se puede acceder al prototipo de pieza en 3D para su descarga e impresión (Figura 16): https://sketchfab.com/3d-models/isinnova-scubamask-adapter-for-respirators-ccff5bd13f524cb49781c9824e21e53d

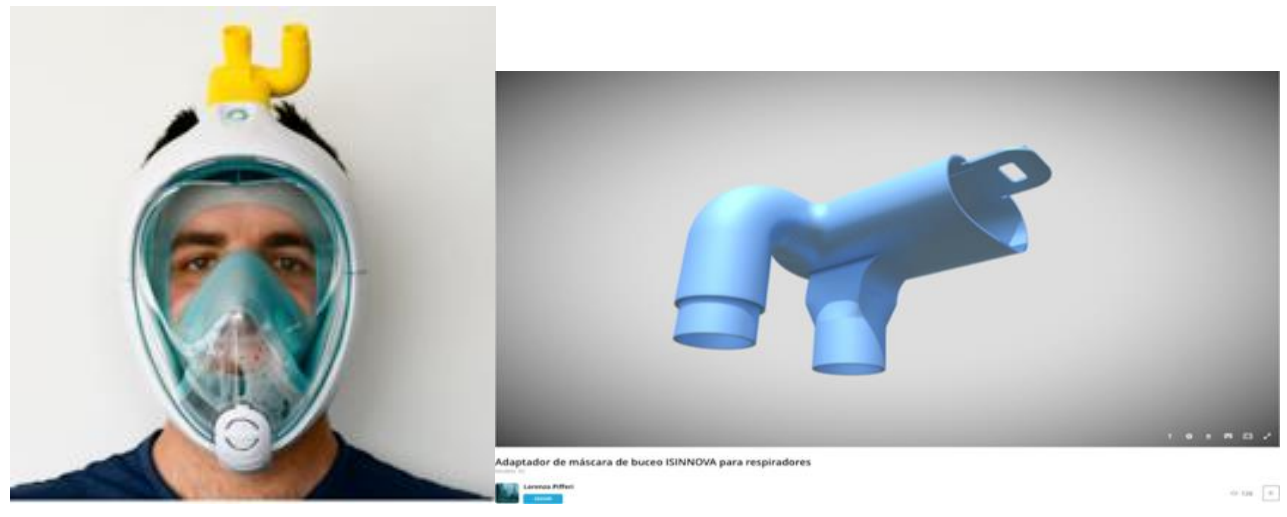

Figura 15. Vista de máscara de buceo snorkel adaptada como respirador a partir de la incorporación de una pieza en 3D que en la foto se muestra de color amarillo. Fuente: Equipo ISINNOVA:

https://www.isinnova.it/easy-covid19/. Figura 16. Vista de pieza en 3D para la conversión de máscaras de buceo snorkel en respiradores.

\subsubsection{Producción de respiradores mediante la técnica de impresión.}

Los respiradores son equipos médicos que permiten respirar a pacientes intubados que se encuentran en la Unidad de Cuidados Intensivos. Debido al estado de emergencia al que se está enfrentando España a causa de la pandemia de COVID-19, el porcentaje de pacientes con síntomas muy graves por los efectos del coronavirus crece exponencialmente. $Y$ ante este crecimiento masivo de pacientes que manifiestan insuficiencia respiratoria, y por tanto, que precisan de asistencia por ventilación mecánica, surge la problemática de la escasez de respiradores artificiales. Y ante esta situación, tiene lugar la iniciativa para la creación de proyectos de código abierto desde una perspectiva makers solidaria por parte de empresas automovilísticas Volkswagen, Seat, Renault, equipos de profesionales de la Escuela de ingenierías Industriales de la Universidad de Málaga y de otras provincias y equipos de centros de investigación que trabajan en el desarrollo de respiradores artificiales a través de la técnica de la impresión en 3D para evitar el colapso de los recursos sanitarios.

\subsubsection{Impresión de mascarillas en 3D.}

El equipo X-Factor mask a través de su página web: http://fabulous.com.co/blog/3d-printed-x-factormask-covid-19-fabulous-tech-center-proposal/ ofrece un prototipo de mascarilla con todos sus accesorios (Figura 17) para su impresión en 3D. Además ha subido el prototipo a la galería de objetos 3D Sketchfab para su descarga, impresión en 3D y fabricación de la mascarilla en 3D: https://sketchfab.com/3d-models/x-factor-mask-covid-19-fabulous$\underline{6333 f 51 \mathrm{fb} 73 \mathrm{a} 4591 \mathrm{ab} 728 \mathrm{c} 936734 \mathrm{ebcf}}$

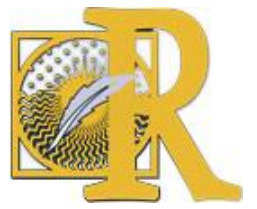




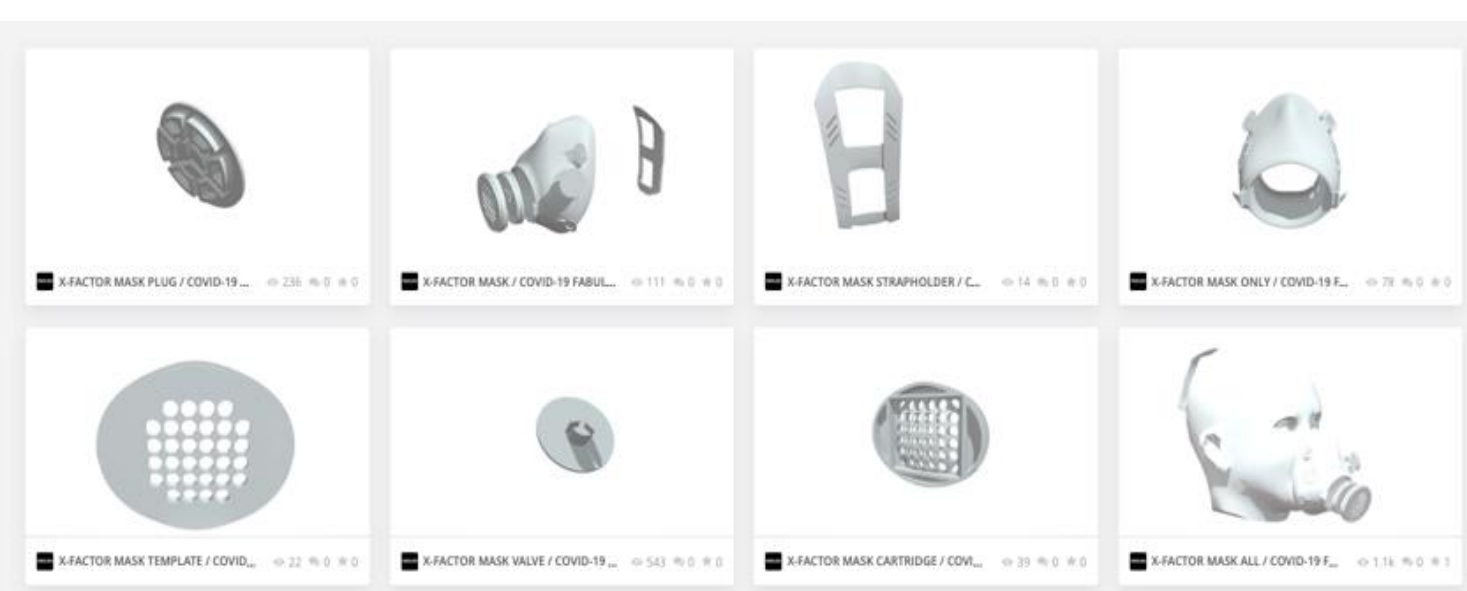

Figura 17. Modelos en 3D de mascarilla y de las piezas que la componen disponibles en la galería de objetos en 3D Sketchfab. Fuente: Equipo X-Factor Mask.

El equipo de Copper3D fabricante del filamento antibacteriano PLAactive ha compartido el diseño de una mascarilla N95 (Figura 18) en formato. stl válido y compatible para su impresión desde cualquier impresora en 3D. Desde su página web el usuario puede descargar el prototipo de modelo en 3D en formato stl para su posterior impresión con impresoras en 3D: https://filament2print.com/es/blog/84 fabricar-mascarilla-impresion-3d.html
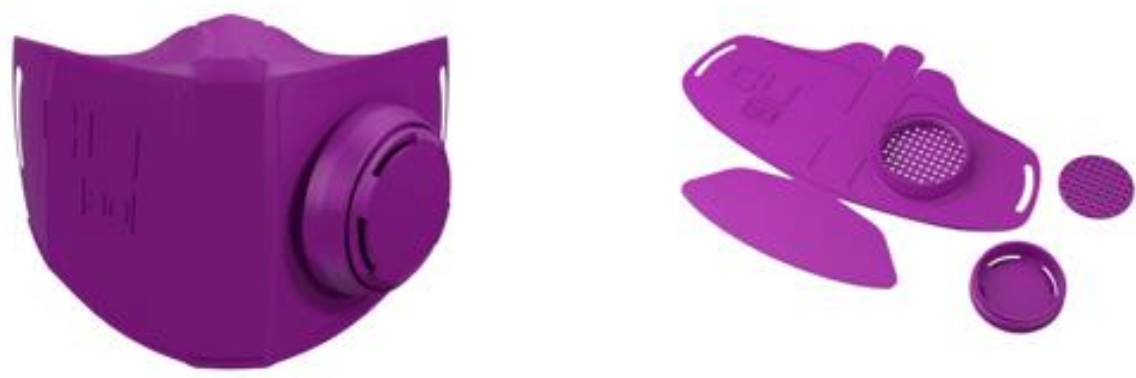

Figura 18. Modelo de mascarilla en 3D impresa. Fuente: Equipo de Copper3D.

\section{Conclusiones.}

Ante una sociedad tan cambiante expuesta a una sucesión de acontecimientos culturales, políticos, ideológicos, económicos, sanitarios y educativos de diversa índole, el desarrollo tecnológico y el desarrollo científico se convierten en una prioridad para dar respuesta eficaz a las demandas y necesidades de los ciudadanos y las ciudadanas para garantizar su seguridad, bienestar y calidad de vida. Así pues, desde una perspectiva conectivista (Siemens, 2004; Downes, 2005; Gutiérrez, 2012) atendiendo a la filosofía colaborativa y creativa del makerspaces (Horizon Report, 2015, 2018) se pretende dar a conocer al profesorado y al alumnado tecnologías emergentes como la realidad aumentada, la realidad virtual, el modelado en 3D y la impresión en 3D para la resolución de

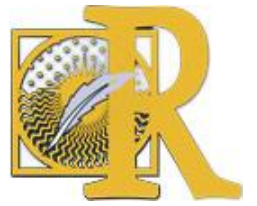


problemas a través del desarrollo de actitudes basadas en la creatividad, la investigación y la colaboración para la comprensión de este fenómeno de emergencia sanitaria y el entrenamiento en fórmulas de actuación a por parte del personal sanitario ante situaciones de emergencia hospitalaria propiciadas por el ingreso masivo de pacientes contagiados por coronavirus a través de la capacitación en escenarios de realidad virtual inmersivos (Moreno y Ramírez, 2016; Moreno, Leiva y Mac, 2017; Moreno, López-Meneses y Leiva, 2018b). Por lo tanto, desde el punto de vista didáctico, pedagógico y procedimental a través del uso de estas tecnologías, a los estudiantes se les otorga un rol más activo y participativo en su propio proceso de aprendizaje mediante la creación de espacios, contenidos, metodologías, materiales y actividades que les permiten un amplio margen de experimentación y manipulación en un sentido más complejo y abstracto para el estudio, el análisis y comprensión de dicho fenómeno de actualidad en cuanto a la naturaleza del virus, su composición genética, su origen, los factores que desencadenan los contagios, la sintomatología que presenta el sujeto infectado y las medidas de prevención y seguridad. De este modo, estos objetos 3D y entornos generados a través de la tecnología de realidad aumentada, realidad virtual, las plataformas y programas de modelado en 3D y la impresión en 3D permiten a los discentes realizar trabajos de investigación interactuando con los elementos de forma virtual y física (Moreno, Leiva y LópezMeneses, 2016; Moreno, López-Meneses y Leiva, 2016; Moreno, López-Meneses y Leiva, 2018a). Asimismo, en las sesiones formativas con el alumnado se posibilita un pensamiento reflexivo, interpretativo y comprensivo sobre la propia práctica acerca de las posibilidades y los beneficios de estas tecnologías aplicadas al ámbito educativo y sanitario. Y por otro lado, se pretende alentar iniciativas y actitudes solidarias impulsando la apertura de recursos informáticos gratuitos mediante la creación de espacios creativos y colaborativos en red desde una perspectiva makerspaces para la democratización del conocimiento y la trasformación social desde un enfoque solidario y moral a través de proyectos de emprendimiento e innovación. De este modo, estos materiales no sólo serán accesibles para el alumnado, el profesorado y las familias de las instituciones educativas, sino que se podrán generalizar, adaptar, modificar y reutilizar para ponerlos a disposición de toda la comunidad social que los precise.

\section{Referencias bibliográficas.}

- Auld, L. (1995). Differences between 3D computing and virtual reality, VR in the Schools. Virtual Reality and Education Laboratory, 1(3). East Carolina University. Greenville, North Carolina USA. Recuperado de: http://vr.coe.ecu.edu/vrits/1-3Auld.htm

- Azuma, R. (1997). A Survey of Augmented Reality. Presence: Teleoperators and Virtual Environments, 6 (4), 355-385.

- Beltrán, P. y Rodríguez, C. (2017). Modelado e impresión en 3D en la enseñanza de las matemáticas: un estudio exploratorio. ReiDoCRea: Revista electrónica de investigación y docencia creativa, 6, 16-28. Recuperado de: http://digibug.ugr.es/bitstream/10481/44193/1/6-2.pdf

- Cabero J. y Barroso J. (2016a). Posibilidades educativas de la realidad aumentada. New Approaches in Educational Research, 5(1), 46-52. doi: 10.7821/naer.2016.1.140

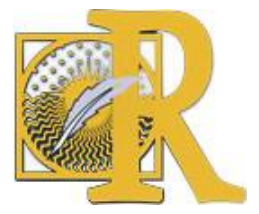


- Cabero, J. y Barroso, J. (2016b). Ecosistema de aprendizaje con realidad aumentada: posibiliades educativas. TCyE: Tecnología, Ciencia y Educación, 5, 141-154. Recuperado de: http://www.tecnologia-ciencia-educacion.com/index.php/TCE/article/view/101

- Cabero, J. y García, F. (coords.) (2016). Realidad aumentada. Tecnología para la formación. Madrid: Síntesis.

- Cabero, J., Leiva, J.J., Moreno, N.M., Barroso, J. y López, E. (2016). Realidad Aumentada y Educación. Innovación en contextos formativos. Barcelona: Octaedro.

- Cabero-Almenara, J., y Ruiz-Palmero, J. (2018). Las Tecnologías de la Información y Comunicación para la inclusión: reformulando la brecha digital. IJERI: International Journal of Educational Research and Innovation, (9), 16-30. Recuperado de https://www.upo.es/revistas/index.php/lJERI/article/view/2665

- Casey, L.L. (1994). Realidad Virtual. Barcelona: McGrawHill.

- Cobo Romaní, C y Moravec, J.W. (2011). Aprendizaje invisible. Hacia una nueva ecología de la educación. Col.lecció Transmedia XXI. Laboratori de Mitjans Interactius. Barcelona: Univesitat de Barcelona.

- Downes, S. (2005). An introduction to Connective Knowledge. [En línea]. Recuperado de: http://www.downes.ca/cgi-bin/page.cgi?post=33034

- Gutiérrez, L. (2012). Coenctivismo como teoría de aprendizaje: conceptos, ideas y posibles limietaciones. Revista Educación y Tecnología, 1, 111-122. Recuperado de: https://dialnet.unirioja.es/servlet/articulo?codigo=4169414

- Horizon Report NMC (2015). Higher Education Edition. Recuperado de: http://www.nmc.org/publication/nmc-horizon-report-2015-higher-education-edition/

- Horizon Report NMC (2018). Higher Education Edition. Recuperado de: https://library.educause.edu/resources/2019/4/2019-horizon-report

- López-Gil, M. y Bernal-Bravo, C. (2019). El perfil del profesorado en la Sociedad Red: reflexiones sobre las competencias digitales de los y las estudiantes en Educación de la Universidad de Cádiz. IJERI: International Journal of Educational Research and Innovation, (11), 83-100.

Recuperado de: https://www.upo.es/revistas/index.php/lJERl/article/view/3265

- Moreno, N.M. y Leiva Olivencia, J.J. (2017). Experiencias formativas de uso didáctico de la realidad aumentada con alumnado del grado de educación primaria en la universidad de Málaga. Revista Edmetic, 6(1), pp. 81-104. Recuperado de: http://www.uco.es/servicios/ucopress/ojs/index.php/edmetic/issue/view/546/showToc

- Moreno, N.M. y Ramírez, M. B. (2016). Uso didáctico de la realidad virtual en los Grados de Educación Infantil y Educación Primaria. En A. Matas Terrón, J.J. Leiva Olivencia, N.M. Moreno Martínez, N. M., A.H. Martín Padilla y E. López Meneses, E. (2016). I Seminario Internacional de Innovación docente e Investigación Educativa. Madrid: Afoe.

- Moreno, N. M., Leiva, J. y López-Meneses, E. (2016). Robótica, modelado 3d y Realidad Aumentada en educación para el desarrollo de las inteligencias múltiples. Aula de Encuentro, 18 (2), 158-183. Recuperado de: https://revistaselectronicas.ujaen.es/index.php/ADE/article/view/3191

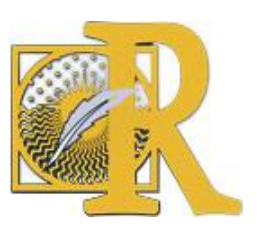


- Moreno, N.M., Leiva, J.J. y López-Meneses, E. (2017). La realidad aumentada como tecnología emergente para la innovación educativa. Revista Notandum. Revista Semestral Internacional de Estudios Académicos, 44-45, 125-140. http://dx.doi.org/10.4025/notandum.44.11

- Moreno, N.M., Leiva, J.J. y Mac, I. (2017). Virtual reality to potenciate the learning scenarios in Higher Education: Experience of educational innovation with the graduate students in criminology. En E. López-Meneses, E., F.M. Sirgnano, M. Reyes Tejedor, M. Cunzio y J. Gómez Galán (Eds.), European innovations in education: Research models and teaching applications (pp. 64-75). Sevilla: AFOE.

- Moreno, N.M., López-Meneses, E. y Leiva, J.J. (2016). Tecnologías emergentes para el desarrollo de la innovación educativa: Modelado en 3D y Realidad Aumentada. En J. Gómez Galán, E. López-Meneses y A.H. Martín Padilla (Eds.), Advances and Innovations in Educational Research (pp.74-83). UMET Press, Universidad Metropolitana, Sistema Universitario Ana G. Méndez, San Juan (Puerto Rico), Sevilla: Editorial AFOE.

- Moreno, N.M., López-Meneses, E. y Leiva, J.J. (2018a). El uso de las tecnologías emergentes como recursos didácticos en ámbitos educativos. International Studies on Law and Education, 29/30. Recuperado de: http://www.hottopos.com/isle29 30/index.htm

- Moreno, N.M., López-Meneses, E. y Leiva, J. J. (2018b). Experiencia universitaria con realidad aumentada y realidad virtual para construir innovación educativa. En E. LópezMeneses, D. Cobos-Sanchiz, A.H. Martín-Padilla, L. Molina García y A. Jaén Martínez (Eds.), Experiencias pedagógicas e innovación educativa. Aportaciones desde la praxis docente e investigadora (pp.1196-1213). Barcelona: Octaedro. Monográfico.

- Nugent, W.R. (1991). Virtual Reality: Advanced Imaging Special Effects Let You Roam in Cyberspace. Journal of the American Society for Information Science, 42(8), 609.

- Portuguez, M. y Gómez, M.G. (2019). Makerspaces como espacios educativos de innovación y desarrollo de emprendimientos. Internacional Journal of Information Systems and Software Engineering for Big Companies (IJISEBC), 6(2),19-32. Recuperado de: https://dialnet.unirioja.es/servlet/articulo?codigo $=7218457$

- Siemens, G. (2004, December). Connectivism: A theory for the digital age. Recuperado de: http://www.itdl.org/Journal/Jan 05/article01.htm

- Smith, A. (2017). Innovación social, democracia y makerspaces. Revista española del tercer sector, 36, 49-74. Recuperado de: https://dialnet.unirioja.es/servlet/articulo?codigo $=6460198$

- Tecnológico de Monterrey (2017). Reporte EduTrends. Radar de Innovación Educativa 2017. Monterrey: Tecnológico de Monterrey.

- Vázquez, E., León, M., Parra, M. E. y López-Meneses, E. (2020). Analysis of Interpersonal Competences in the Use of ICT in the Spanish University Context. Sustainability, 12(2), 476. Recuperado de: https://doi.org/10.3390/su12020476

- Vera, G., Ortega, J.A. y Burgos, M.A. (2003). La realidad virtual y sus posibilidades didácticas. Etic@net, 2 (en línea). Granada. Recuperado de: http://www.ugr.es/ sevimeco/revistaeticanet/Numero2/Articulos/Realidadvirtual.pdf

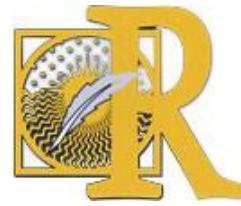




\section{Webgrafía.}

- Sitios web de recursos informáticos, proveedores y diseñadores citados en el trabajo:

- 3D Warehouse: https://3dwarehouse.sketchup.com/?hl=es

- 3DC Education: https://3dc.io

- Archive 3D: https://archive3d.net

- Blender: https://www.blender.org/

- Comunidad Cults: https://cults3d.com/es/modelo-3d/casa/vinchas-para-mascara-protectora-convisera-y-sin-visera-varios-modelos-seguridad-corona-virus

- Cubo Merge: https://mergeedu.com/download/file.php?f=paper-merge-cube.pdf

- Equipo Copper3D filament2print: https://filament2print.com/es/blog/84 fabricar-mascarillaimpresion-3d.html

- Equipo ISINNOVA: https://www.isinnova.it/easy-covid19-esp-2/

- Equipo X-Factor Mask: http://fabulous.com.co/blog/3d-printed-x-factor-mask-covid-19-fabuloustech-center-proposal/

- Google Poly: https://poly.google.com

- Induna-art-design: http://www.induna.ca

- Sketchfab: https://sketchfab.com/feed

- SketchUp: http://www.sketchup.com/es

- Tinkercad Autodesk: https://www.tinkercad.com

- UPShield Face Shield Mask: https://lanpdt.life/upshield

- Umbral Virtual: Capacitación en realidad virtual sobre el COVID by Angelo RV (28 de mayo de 2020): $\quad$ http://umbralvirtual.com/2020/05/28/capacitacion-en-realidad-virtual-sobre-el-covid19/?fbclid=IwAR2IKHkzCfUhghsAB7mYdGLXnenFt6DNw69HQdGkbhOrkkwjkrqV0aY0d3A 